\title{
Shared neighborhood effects in masked orthographic priming
}

\author{
WALTER J. B. VAN HEUVEN and TON DIJKSTRA \\ University of Nijmegen, Nijmegen, The Netherlands \\ JONATHAN GRAINGER \\ CNRS and Université de Provence, Aix-en-Provence, France
}

and

HERBERT SCHRIEFERS

University of Nijmegen, Nijmegen, The Netherlands

\begin{abstract}
Several studies have found effects of orthographically related masked nonword primes on lexical decisions to target words. These effects have been explained by the neighborhood characteristics of the target word (Forster, 1987), but the neighborhood characteristics of the prime in combination with the target are also found to be important (Hinton, Liversedge, \& Underwood, 1998). In this study, we present a new account of masked form-priming effects based on the shared neighborhood of prime and target. Shared neighbors are words that are activated by both prime and target. According to the interactive activation model (McClelland \& Rumelhart, 1981), shared neighborhood determines the size of priming effects. This prediction was tested and confirmed in a masked priming experiment that manipulated the shared neighborhood density of complete primes.
\end{abstract}

The study of visual word recognition in the last three decades has shown that target word processing can be influenced by a previously presented letter string (the prime). Since the beginning of the eighties, a variant of this priming paradigm, the masked priming paradigm, has become very popular. The masked priming presentation sequence starts with a mask (usually a row of hash marks), followed by a very brief presentation of the prime in lowercase and then the target string in uppercase. Owing to the short prime presentation and the presence of a mask, participants are unaware of the prime. In contrast to unmasked priming, this procedure discourages the use of strategies participants might adopt on detecting any relation between primes and targets (Forster \& Davis, 1984; Forster, Davis, Schoknecht, \& Carter, 1987). The masked priming technique has been applied fruitfully to investigate many aspects of visual word recognition. In this study, we will use it to examine how target word recognition is affected by the combined orthographic characteristics of masked nonword primes and the targets themselves.

The research reported here was supported by a grant from the NFS Fund for Dutch-French collaboration (Netherlands Organization for Scientific Research, NWO, The Netherlands) awarded to the Nijmegen Institute for Cognition and Information (NICI). We thank Ken Paap, Keith Rayner, an anonymous reviewer, and especially Ken Forster for their helpful comments. Correspondence concerning this article should be sent to W. J. B. van Heuven, NICI, University of Nijmegen, Montessorilaan 3, 6525 HR, Nijmegen, The Netherlands (e-mail: heuven@ nici.kun.nl).
The masked priming technique was used by Forster and Davis (1984) to investigate the effect of orthographic relatedness on target word processing in a lexical decision task. They found no response time (RT) differences for orthographically related word primes (e.g., lack-LOCK) relative to unrelated primes (e.g., bowl-LOCK). However, in a later study by Forster et al. (1987), facilitation effects were obtained (Experiment 1) for target items preceded by transposition (anwser-ANSWER) or substitution (antwerANSWER) nonword primes, relative to unrelated primes. This result, which clearly deviated from that obtained by Forster and Davis (1984), was explained by the authors in terms of stimulus length. Whereas Forster and Davis (1984) used only four-letter words, the average word length in the first experiment by Forster et al. (1987) was 7.5 letters. This length hypothesis was tested in a second experiment that involved words of different lengths. Reliable priming effects were observed for combinations of substitution primes and target words that were eight letters long, but no priming effects occurred when such primetarget combinations consisted only of four letters.

Forster et al. (1987) related the apparent lengthdependent effect of form priming to differences in neighborhood density between long and short target words. Neighbors are words that can be derived from a given target word by changing one letter, and neighborhood density refers to the number of such neighbors for a particular target word (Coltheart, Davelaar, Jonasson, \& Besner, 1977). Whereas longer words generally have few neighbors, short words often have many neighbors (see also Frauenfelder, Baayen, Hellwig, \& Schreuder, 1993). 
Forster et al. argued that the size of the target word neighborhood is responsible for the priming effect, rather than the target word's length. They tested this hypothesis with prime-target pairs, consisting of four letters, which were drawn from either a low- or a high-density neighborhood (Experiment 6). Orthographically related primes facilitated target word recognition only when the prime-target pair was drawn from a low-density neighborhood.

Because in this study primes and targets were drawn from the same type of neighborhood, it remains unclear whether, apart from the characteristics of the target neighborhood, the characteristics of the prime neighborhood also affected form-priming effects. In a further masked prime lexical decision experiment, Forster (1987) investigated the role of prime neighborhood density. In this experiment, he used four-letter target words that had a large number of neighbors and primed these targets with two types of orthographically related nonword primes and a control prime. The two related nonword primes had either a low or a high number of neighbors. No priming effect was found for the two orthographically related nonword primes, and there was no difference between the primes with a low- or high-density neighborhood. Forster concluded that only the neighborhood density of the target determines whether priming is obtained or not. This conclusion was referred to as the "density constraint on form priming" by Forster and Davis (1991).

However, a recent study indicates that the orthographic characteristics of partial nonword primes determine the size of the priming effect in combination with the target characteristics. Hinton, Liversedge, and Underwood (1998) investigated the effects of partial primes on target word recognition in a lexical decision task. In their first experiment using unmasked primes presented for $100 \mathrm{msec}$, partial primes were constructed by removing one letter from the target word (e.g., partial prime ath, target word PATH). In one type of partial prime, a letter was missing at a position at which several words could be formed. For example, from the prime ath, several words could be derived by filling in letters at the missing onset letter position (e.g., MATH, BATH). This type of partial prime was called the ambiguous prime. Another prime type, the unambiguous prime, was constructed by removing a letter at a position at which only the target word could be derived (e.g., partial prime $p a \_h$ ). Only unambiguous primes were found to facilitate target word processing. The same pattern of results was obtained in a second masked priming experiment with a new set of stimuli. In this second experiment, a prime duration of $60 \mathrm{msec}$ was used, and the missing letter in the primes was replaced by a percent sign (e.g., \%ond).

Theoretically, masked orthographic priming results have been interpreted in terms of two classes of models. One class of models is based on the search metaphor (Forster, 1976), and the other on a parallel activation approach, exemplified by the interactive activation (IA) model (McClelland \& Rumelhart, 1981). The most recent version of the search model is the entry-opening model (Forster, 1998; Forster \& Davis, 1984). According to this model, lexical access involves a two-stage filtering process. In the first stage (called fast search), a number of lexical entries are opened when they closely match the input letter string. In the second stage (called the evaluation process), the proposed lexical candidates are matched to the stimulus input, the final candidate is selected, and all other entries are closed down. An important assumption is that the masked priming procedure does not close down the entries opened by the prime. Masked priming effects can now be explained by assuming that the prime already opens the target entry, which allows a faster evaluation of the subsequently presented target.

However, it seems that the entry-opening model cannot explain the results of Hinton et al. (1998). Because the ambiguous and unambiguous partial primes are both closely matched to the target string, they would both open the lexical entry of the target item. As was argued by Hinton et al. (1998, pp. 1301-1302), this would result in a similar facilitation effect for ambiguous and unambiguous partial primes. ${ }^{1}$ However, Hinton et al.'s data showed that only unambiguous primes facilitated target word recognition.

Can the data from Hinton et al. (1998) be accounted for in terms of the alternative IA approach? Two characteristics of the IA model are relevant for this question. First, in the model, both the target word and all its neighbors are activated upon presentation of the target letter string. Analogously, when a nonword string is presented to the model, all word neighbors of the nonword become active (of course, the nonword itself does not have a lexical representation). A second relevant characteristic of the IA model is the lateral inhibition mechanism. Because of this mechanism, activated word neighbors inhibit the target word and slow down its recognition process. During the masked priming procedure, the prime and target stimuli cause significant activation in word representations that are orthographic neighbors of these stimuli. Word representations that are strongly activated by both the prime and the target will have the greatest inhibitory influence during target processing. The finding by Hinton et al. that only unambiguous primes facilitate target word recognition can be explained by the IA model as a consequence of target preactivation in the absence of shared neighbors. In the case of ambiguous primes, similar facilitation effects arise, but these are canceled out by effects of lateral inhibition owing to the presence of active shared neighbors. As a consequence, no priming effects are observed for ambiguous primes.

The IA model predicts that shared neighborhood (henceforth, SN) will influence the target word recognition process also when complete nonword primes (as opposed to partial primes) are presented. For example, the Dutch target word DOLK can be primed by two types of orthographically related nonwords, exemplified by HOLK and DOLS. The nonword prime HOLK has six Dutch word neighbors-DOLK, HOEK, KOLK, VOLK, WOLK, and TOLK- 


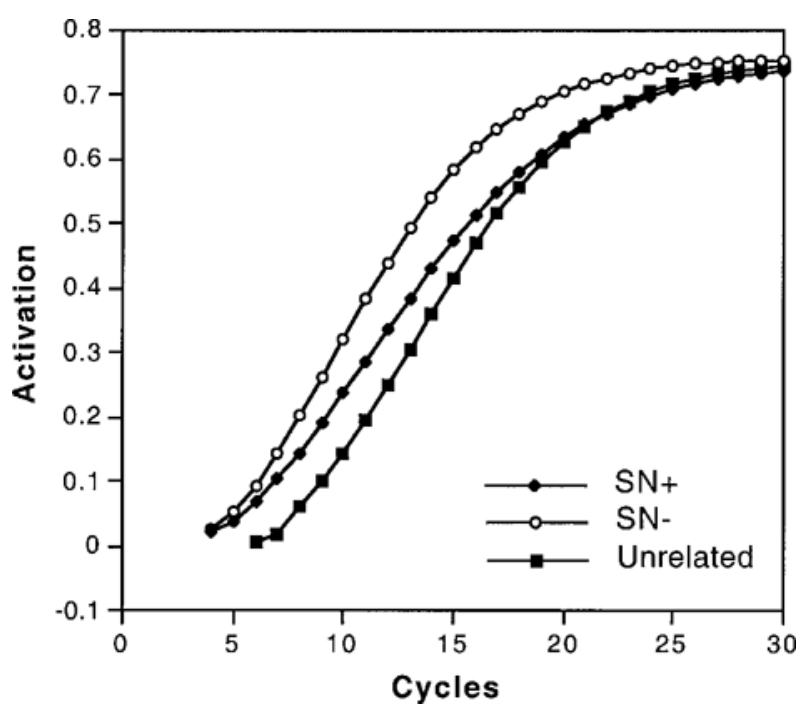

Figure 1. Mean activation curve of the word targets preceded by three prime types: $\mathrm{SN}+$ (prime-target pair with shared neighbors), $\mathrm{SN}$ - (prime-target pair with no shared neighbors), and unrelated (prime-target pair without form overlap). The primes were presented to the model on the first two cycles, and the targets on the third cycle until cycle 30 .

whereas the target word DOLK has the following five neighbors: DOEK, KOLK, VOLK, WOLK, and TOLK. Thus, there are four neighbors shared by prime HOLK and target DOLK - namely, KOLK, VOLK, WOLK, and TOLK. In contrast, no word neighbors are shared by prime DOLS and target DOLK. As a consequence, the nonword prime DOLS should facilitate the target word DOLK, whereas the nonword prime HOLK should not. As we will see in the Method section, simulations with the IA model confirm these informal predictions. Thus, complete primes should result in a target data pattern similar to that caused by the partial primes used by Hinton et al. (1998). We will test this prediction in a lexical decision task with masked primes that manipulates the number of neighbors shared by prime and target. In addition, we will investigate whether the size of the $\mathrm{SN}$ effect depends on prime duration.

\section{METHOD}

\section{Participants}

Sixty students of the University of Nijmegen participated in this study, 30 in each of the two prime duration conditions (30 and $60 \mathrm{msec})$. All the students were native speakers of Dutch with normal or corrected-to-normal vision.

\section{Stimuli and Design}

The target items consisted of 18 words and 18 nonwords, all four letters in length. The word targets were selected from a list of 1,284 Dutch four-letter lexicon extracted from the CELEX database (Baayen, Piepenbrock, \& van Rijn, 1993). This lexicon consisted of words with a printed frequency of at least one occurrence per million (opm). The target words were all nouns with a low frequency (mean, $4.2 \mathrm{opm}$ ) and a low-density neighborhood (mean, 4.1 neighbors). All the target words were monosyllabic words with a CCVC, CVCC, or CVVC structure. For each target word, three types of nonword primes with a similar structure were constructed. Two types of nonword primes were orthographically related with the corresponding target (differing by only one letter), and the third type was orthographically unrelated. The two types of orthographically related nonword primes differed in terms of the word neighbors they shared with the target word. One type of prime ( $\mathrm{SN}-$ ) shared no neighbors with the target word (e.g., dols-DOLK), whereas the other type $(\mathrm{SN}+)$ shared one or more neighbors with the target word (e.g., holk-DOLK, with shared neighbors KOLK, VOLK, WOLK, and TOLK). The average number of shared neighbors in the $\mathrm{SN}+$ condition was 2.6 words. Both prime types were matched on the letter position at which the nonword prime differed from the target word ( $\mathrm{SN}+$, mean letter position of $2.3, \mathrm{SD}$ of 1.4 ; $\mathrm{SN}-$, mean position of 2.6, $S D$ of 1.4). The complete list of the prime-target pairs can be found in the Appendix.

The word material was also run through simulations in order to check whether the IA model could predict the differences between the prime types. In the simulation, every prime-target pair was presented to the model. The prime string was presented on the first and second cycle, and at the third and following cycles the target string was presented until the model had been running for 30 cycles. After running simulations with all the prime-target pairs, the average target node activation curve for each of the three prime types was calculated. The resulting activation curves in Figure 1 show that SNprime types generally lead to higher activation levels of the target than do the unrelated prime types. Thus, the model predicts that $\mathrm{SN}-$ prime types will lead to a facilitation effect. According to the simulations, there are no differences in the activation of the target after $\mathrm{SN}+$ and unrelated prime types late in processing, whereas early in processing the $\mathrm{SN}+$ prime types lead to a higher activation of the target than do the unrelated prime types. However, the order of prime types in terms of relative degree of target activation is always $\mathrm{SN}-, \mathrm{SN}+$, unrelated prime type. Thus, according to the model, $\mathrm{SN}+$ primes should lead either to no effect or to a facilitation effect if a decision is made early in processing.

To construct the nonword targets, a new list of 18 nouns was selected from the same lexicon as that described before. One letter was changed from these words to construct nonword targets. Next, three types of nonword primes were constructed for these targets with a similar manipulation as for the word targets.

The 36 prime-target pairs (18 pairs with word targets and 18 pairs with nonword targets) were rotated across all priming conditions over three groups of participants. At the same time, the three priming conditions were also rotated over three blocks within participants. Each target was preceded by three different primes in different blocks for the same participant. In each block, the participant encountered all the prime types. Thus, each participant saw 36 prime-target pairs in each block, 6 from each prime type. Across blocks, each participant saw all the prime-target pairs. Furthermore, half of the participants were allocated to a short prime duration condition $(30 \mathrm{msec})$ and the other half to a long prime duration condition $(60 \mathrm{msec})$.

\section{Procedure}

A trial started with a row of four hash marks (\#\#\#\#) as mask, presented for $510 \mathrm{msec}$ (34 screen refreshes). The mask was immediately followed by the prime presented in lowercase for $30 \mathrm{msec}(2$ screen refreshes) in the short prime duration condition or $60 \mathrm{msec}$ (4 screen refreshes) in the long prime duration condition. Next, the prime was replaced by the target presented in uppercase. The target remained on the screen until the participant pressed a button or for $2,000 \mathrm{msec}$. After a blank intertrial interval of $1,000 \mathrm{msec}$, the next trial started. The timing of the mask, prime, and target presentation was synchronized with the refresh rate of the monitor $(\mathrm{a} 67-\mathrm{MHz}$ Apple 14-in. Trinitron monitor).

Letter strings were presented in black letters (Courier 24 points) at the center of the screen on a white background. The participants were instructed to make a lexical decision on the letter strings ap- 
Table 1

Mean Response Times (RTs, in Milliseconds), Standard Errors (SE), and Error Percentages (E\%) for Word Targets Preceded by

Different Types of Nonword Primes in Each Block and Prime Duration

\begin{tabular}{|c|c|c|c|c|c|c|c|c|c|c|c|}
\hline \multirow[b]{2}{*}{ Prime Duration } & \multicolumn{3}{|c|}{$\mathrm{SN}+$} & \multicolumn{3}{|c|}{$\mathrm{SN}-$} & \multicolumn{3}{|c|}{ Unrelated } & \multicolumn{2}{|c|}{ Effect } \\
\hline & $M$ & $S E$ & $\mathrm{E} \%$ & $M$ & $S E$ & $\mathrm{E} \%$ & $M$ & $S E$ & $\mathrm{E} \%$ & $\mathrm{SN}+$ & $\mathrm{SN}-$ \\
\hline \multicolumn{12}{|l|}{$30 \mathrm{msec}$} \\
\hline Block 1 & 583 & 16.2 & 5.0 & 555 & 12.9 & 2.8 & 579 & 13.8 & 7.2 & -4 & 24 \\
\hline Block 2 & 513 & 11.5 & 2.2 & 504 & 9.3 & 2.8 & 525 & 9.3 & 4.4 & 12 & 21 \\
\hline Block 3 & 504 & 12.3 & 2.8 & 497 & 11.5 & 1.7 & 532 & 11.2 & 1.1 & 28 & 35 \\
\hline \multicolumn{12}{|l|}{$60 \mathrm{msec}$} \\
\hline Block 1 & 590 & 13.1 & 4.4 & 567 & 14.7 & 3.3 & 602 & 16.0 & 5.0 & 12 & 35 \\
\hline Block 2 & 537 & 17.9 & 1.7 & 519 & 13.1 & 0.6 & 537 & 11.5 & 0.6 & 0 & 28 \\
\hline Block 3 & 510 & 11.7 & 1.7 & 503 & 11.7 & 1.1 & 539 & 13.8 & 3.3 & 29 & 36 \\
\hline \multicolumn{12}{|l|}{ All blocks } \\
\hline $30 \mathrm{msec}$ & 533 & 11.0 & 3.4 & 518 & 10.0 & 2.4 & 545 & 9.7 & 4.3 & 12 & 27 \\
\hline $60 \mathrm{msec}$ & 546 & 11.5 & 2.6 & 529 & 11.8 & 1.7 & 559 & 12.0 & 3.0 & 13 & 30 \\
\hline Overall & 540 & & & 524 & & & 552 & & & 12 & 28 \\
\hline
\end{tabular}

Note-Priming effect is reported as a positive value.

pearing in uppercase. They were not informed about the primes. The participants were instructed to make their decisions as quickly as possible without making errors. Before the experiment, the participants received a series of 18 practice trials of the same type as those in the experiment. Next, the three test blocks were presented, separated by short breaks. Each block started with 2 filler trials. In total, the experiment comprised 114 prime-target pairs.

\section{RESULTS}

Erroneous responses (3.5\%) and RTs that were outside the range of two standard deviations of a participant's and item's mean $(0.2 \%)$ were omitted from the analysis. The RTs of word targets were submitted to an analysis of variance (ANOVA), with block (first, second, or third) and prime type ( $\mathrm{SN}+, \mathrm{SN}-$, and unrelated) as within-subjects factors and prime duration ( 30 or $60 \mathrm{msec}$ ) as a betweensubjects factor. The overall RTs for words and nonwords were 539 and $607 \mathrm{msec}$, respectively, with corresponding error percentages of $1.4 \%$ and $2.0 \%$. Table 1 gives the mean RTs for word targets in the different test conditions.

The analysis showed a main effect of prime type $\left[F_{1}(2,116)=25.68, p<.001 ; F_{2}(2,68)=20.27, p<.001\right]$ and a main effect of block $\left[F_{1}(2,166)=78.90, p<.001\right.$; $\left.F_{2}(2,68)=124.67, p<.001\right]$. The interaction between these factors was not significant $\left[F_{1}(4,232)=1.86, p=.12\right.$; $\left.F_{2}(4,136)=2.00, p=.10\right]$. There was no effect of prime duration $\left[F_{1}(1,58)<1 ; F_{2}(1,34)=2.03, p=.16\right]$, and this factor did not interact with prime type or block (all $F_{\mathrm{S}}<1$ ).

Planned comparisons showed that target words preceded by $\mathrm{SN}-$ primes were significantly $(28 \mathrm{msec})$ faster than those preceded by unrelated primes $\left[F_{1}(1,59)=55.57\right.$, $\left.p<.001 ; F_{2}(1,17)=39.68, p<.001\right]$. Target words preceded by $\mathrm{SN}+$ primes were reacted to $12 \mathrm{msec}$ faster than those preceded by unrelated primes $\left[F_{1}(1,59)=9.87, p<\right.$ $\left..01 ; F_{2}(1,17)=7.85, p<.01\right]$. Furthermore, there was a significant RT difference of $16 \mathrm{msec}$ between the $\mathrm{SN}+$ and the $\mathrm{SN}-$ prime types $\left[F_{1}(1,59)=14.3, p<.001 ; F_{2}(1,17)=\right.$ $15.18, p<.001]$.
The error data showed no effect of prime type $\left[F_{1}(2,116)\right.$ $\left.=1.57, p=.21 ; F_{2}(1,34)=2.18, p=.30\right]$, but the effect of block was significant $\left[F_{1}(2,116)=9.91, p<.001\right.$; $\left.F_{2}(2,68)=9.57, p<.001\right]$. No effect of prime duration was found $\left[F_{1}<1 ; F_{2}(1,34)=1.12, p=.30\right]$. None of the interactions between the three factors was significant.

Even though the interaction between block and prime type did not reach significance, Table 1 suggests a gradual increase of the effect (in RTs) of the SN+ condition relative to the unrelated condition across blocks. We therefore conducted an ANOVA over the RTs of the first presentation block only, with the factors prime type as a within-subjects factor and prime duration as a betweensubjects factor. The mean RTs were $587 \mathrm{msec}$ for $\mathrm{SN}+$, $561 \mathrm{msec}$ for $\mathrm{SN}-$, and $591 \mathrm{msec}$ for the unrelated prime types. The effect of prime type was significant $\left[F_{1}(2,188)=\right.$ $\left.7.64, p<.01 ; F_{2}(2,70)=6.59, p<.01\right]$. Planned comparisons indicated that RTs to targets preceded by $\mathrm{SN}-$ primes were significantly faster $(30 \mathrm{msec})$ than those to targets preceded by unrelated prime types $\left[F_{1}(1,59)=\right.$ $\left.13.63, p<.001 ; F_{2}(1,35)=13.02, p<.01\right]$. In contrast with the analysis across blocks, this analysis revealed no difference between $\mathrm{SN}+$ and unrelated primes (both $\left.F_{\mathrm{s}}<1\right)$. In addition, the $\mathrm{SN}+$ prime type was significantly slower $(26 \mathrm{msec})$ than the $\mathrm{SN}-$ prime type $\left[F_{1}(1,59)=7.80, p<.01 ; F_{2}(1,35)=8.8, p<.01\right]$.

\section{DISCUSSION}

In this study, we investigated the effects of shared neighbors in a lexical decision task with masked primes. On the basis of simulations with the IA model (see Figure 1), we predicted that prime-target pairs with no shared neighbors $(\mathrm{SN}-$ ) would show a priming effect, relative to unrelated prime-target pairs (e.g., RTs to dols-DOLK would be faster than wers-DOLK). The primetarget pairs with shared neighbors $(\mathrm{SN}+)$, however, should show either no priming effect or a small facilita- 
tion effect if a decision is made early in processing (e.g., holk-DOLK vs. wers-DOLK).

In accordance with these predictions, RTs to word targets were faster when preceded by $\mathrm{SN}-$ primes than when preceded by unrelated primes. The RTs to targets preceded by $\mathrm{SN}+$ primes also showed a relative priming effect, but this effect was much smaller than that for the $\mathrm{SN}-$ primes. As can be seen in Figure 1, this is exactly the pattern predicted by the IA model under the assumption that a decision was made in the early stages of processing (fast reactions or low activation threshold for lexical selection; see Forster \& Veres, 1998, for a similar presentation of activation plots). In this context, we must note that the experimental design involved a repetition of each target item in three blocks. Since there is currently no mechanism to simulate this repetition effect in the IA model, it would probably be more appropriate to compare the simulation data with the RT data of the first presentation block only. Relative to the unrelated prime type, the $\mathrm{SN}+$ prime type resulted in RTs that were a nonsignificant $8 \mathrm{msec}$ faster, whereas the $\mathrm{SN}-$ prime type was significantly faster by $30 \mathrm{msec}$. These results are completely in line with the present simulations if one assumes a selection threshold of 0.67 , as is often done in simulations with the IA model (e.g., Jacobs \& Grainger, 1992).

In sum, the present study showed that $\mathrm{SN}$ between prime and target is an important factor determining the speed of target word processing in masked priming. The empirical results were completely consistent with the IA model's predictions. ${ }^{2}$ The IA model was able to predict these results because of a mechanism that is basic to its architecture-namely, lateral inhibition. Although both types of primes used in the present study preactivated the target, inducing a facilitation effect, only the $\mathrm{SN}+$ prime type simultaneously activated shared neighbors. According to the IA model, these shared neighbors significantly slow down target word processing through lateral inhibition. As a consequence, the model predicted no priming effect for these $\mathrm{SN}+$ primes. As was pointed out in the introduction, search models without an adequate specification of the lexical competition mechanism cannot account for the present effects of SN.

The notion of shared neighbors not only accounts for the present data and those by Hinton et al. (1998), but also can be applied to reinterpret established findings from the literature. The density constraint (Forster \& Davis, 1991) can be reformulated as an SN effect. If a target word has a large neighborhood, there is a greater chance that prime and target share neighbors, resulting in the absence of a priming effect. Furthermore, it is less likely that a prime preceding a target word with a low-density neighborhood has any neighbors in common with the target, thus increasing the likelihood of facilitation in target word processing, relative to an unrelated prime. Because the density constraint itself was a reformulation of the length-dependent effect of form priming (Forster
\& Davis, 1984), the new notion of SN can be used as one common explanation for the relevant data on form priming with nonword primes. ${ }^{3}$

As an example of the role of shared neighbors in masked lexical decision, we also examined the null effect observed in Experiment 4 of Lukatela, Frost, and Turvey (1998). These authors found that an orthographically related prime that shared the onset with a highdensity target word did not prime that target. Thus, clep did not prime CLIP relative to the unrelated prime prem. An inspection of their stimuli indicated that 27 out of 48 prime-target pairs had one or more shared neighbors. We divided the prime-targets pairs from their study into two groups, one with shared neighbors and one without shared neighbors. The mean RTs for these groups revealed a relative facilitation effect of $22 \mathrm{msec}$ for primes that shared no neighbors with the target and a relative inhibition effect of $10 \mathrm{msec}$ for primes that shared one or more neighbors with the target. Thus, whereas Lukatela et al. explained the failure to observe a facilitation effect as being due to a missing rime, our analysis indicates that the presence of shared orthographic neighbors may be responsible for the absence of a priming effect.

The notion of shared neighbors can also be extended to studies that used orthographic word primes, rather than nonword primes, in masked lexical decision. A word prime can be seen as the most activated shared neighbor of the target word, which, according to the IA model, should even induce an inhibition effect as a consequence of strong lexical competition. Such an inhibition effect was indeed obtained in a study of Segui and Grainger (1990). For instance, the RT to a low-frequency French target word CHAR preceded by a masked high-frequency French word neighbor chat was slowed down, relative to an unrelated prime condition. The IA model was able to simulate this inhibitory priming effect (Jacobs \& Grainger, 1992). ${ }^{4}$

Considering the present study in relation to earlier studies, there is one aspect of our data that deserves attention. We observed a small facilitation effect of $\mathrm{SN}+$ primes, whereas Hinton et al. (1998) did not find any effect of such primes (the ambiguous primes in their study). Explanations for this discrepancy can appeal to subtle differences in prime characteristics, target characteristics, or experimental design between the two studies. However, as was noted above, our facilitation effect for $\mathrm{SN}+$ primes disappears if one considers only the first presentation of the target word. Thus, one possibility is that target word repetition underlies the observed facilitation effect. For the first block of our study, the data pattern is analogous to that of Hinton et al.

In conclusion, $\mathrm{SN}$, as introduced in this study, turns out to be an important determinant of the effect of orthographically related primes on target words. The results of the present experiment and simulation also indicate that, within the theoretical framework of the IA model, lateral inhibition is a crucial mechanism to account for masked priming effects. 


\section{REFERENCES}

ANDrews, S. (1997). The effect of orthographic similarity on lexical retrieval: Resolving neighborhood conflicts. Psychonomic Bulletin \& Review, 4, 439-461.

BaAyen, H., Piepenbrock, R, \& VAn Rijn, H. (1993). The CELEX lexical database [CD-ROM]. Philadelphia: University of Pennsylvania, Linguistic Data Consortium.

Coltheart, M., Davelaar, E., Jonasson, J. T., \& Besner, D. (1977). Access to the internal lexicon. In S. Dornic (Ed.), Attention and performance VI (pp. 535-555) New York: Academic Press.

Forster, K. I. (1976). Accessing the mental lexicon. In R. J. Wales \& E. W. Walker (Eds.), New approaches to language mechanisms (pp. 257-287). Amsterdam: North-Holland.

FoRSTER, K. I. (1987). Form-priming with masked primes: The best match hypothesis. In M. Coltheart (Ed.), Attention and performance XII: The psychology of reading (pp. 127-146). Hillsdale, NJ: Erlbaum.

FORSTER, K. I. (1998). The pros and cons of masked priming. Journal of Experimental Psycholinguistic Research, 27, 203-233.

Forster, K. I., \& DAVIS, C. (1984). Repetition priming and frequency attenuation in lexical access. Journal of Experimental Psychology: Learning, Memory, \& Cognition, 10, 680-698.

Forster, K. I., \& DAVIS, C. (1991). The density constraint on formpriming in the naming task: Interference effects from a masked prime. Journal of Memory \& Language, 30, 1-25.

Forster, K. I., Davis, C., Schoknecht, C., \& Carter, R. (1987). Masked priming with graphemically related forms: Repetition or partial activation? Quarterly Journal of Experimental Psychology, 39A, 211-251.

Forster, K. I., \& Veres, C. (1998). The prime lexicality effect: Formpriming as a function of prime awareness, lexical status, and discrimination difficulty. Journal of Experimental Psychology: Learning, Memory, \& Cognition, 24, 498-514.

Frauenfelder, U. H., BaAyen, R H., Hellwig, F. M., \& Schreuder, R. (1993). Neighborhood density and frequency across languages and modalities. Journal of Memory \& Language, 32, 781-804.

Hinton, J., Liversedge, S. P., \& Underwood, G. (1998). Neighborhood effects using a partial priming methodology: Guessing or activation? Journal of Experimental Psychology: Learning, Memory, \& Cognition, 24, 1294-1305.

JACOBS, M. A., \& GRAINGER, J. (1992). Testing a semistochastic variant of the interactive activation model in different word recognition experiments. Journal of Experimental Psychology: Human Perception \& Performance, 18, 1174-1188.

Lukatela, G., Frost, S. J., \& Turvey, M. T. (1998). Phonological priming by masked nonword primes in the lexical decision task. Journal of Memory \& Language, 39, 666-683.

McClelland, J. L., \& Rumelhart, D. E. (1981). An interactive activation model of context effects in letter perception: Pt. 1. An account of basic findings. Psychological Review, 88, 375-405.

Segui, J., \& GRAInger, J. (1990). Priming word recognition with orthographic neighbors: Effects of relative prime-target frequency. Journal of Experimental Psychology: Human Perception \& Performance, 16, 65-76.

Ziegler, J. C., \& Perry, C. (1998). No more problems in Coltheart's neighborhood: Resolving neighborhood conflicts in the lexical decision task. Cognition, 68, B53-B62.

\section{NOTES}

1. Of course, the entry-opening model might be able to account for the results of Hinton et al. (1998) if some additional but as yet unspecified mechanisms were introduced.

2. Because Dutch has a transparent spelling system with a high correlation between orthography and phonology, we cannot exclude that phonological factors contributed to the present data pattern. However, our simulations indicate that a purely orthographic model, such as the IA model, can adequately account for the present results.

3. Because Forster (1987) found no effects of prime neighborhood, we focused on the combined neighborhood characteristics of prime and target. However, prime neighborhood density itself was not controlled for, implying a possible confound in our materials. Indeed, when we computed the average number of neighbors in the three prime conditions, we found that these were not matched $(\mathrm{SN}+, 6.1$; $\mathrm{SN}-, 3.4$; unrelated, 6.1 neighbors). To test whether this factor affected our results, we divided the prime-target pairs into two groups of nine target items that were matched or not across the three conditions in terms of prime neighborhood density. An analysis with this additional between-items factor, prime neighborhood, revealed no significant effect of this factor (both $F \mathrm{~s}<1$ ) and no significant interaction with any of the other factors (all $F \mathrm{~s}<1$ ). Thus, the same pattern of results, showing the effect of SN, was obtained irrespective of prime neighborhood density. This finding supports Forster's (1987) conclusion that prime neighborhood density itself is not a major factor in masked orthographic priming.

4. It should be noted that manipulations of neighborhood density in unprimed lexical decision experiments often give rise to facilitatory effects of this variable (see Andrews, 1997, for a review). This might be due to between-items comparisons used in these studies that allow for possible contamination from uncontrolled variables (e.g., Ziegler \& Perry, 1998), a problem that the use of a priming paradigm partly alleviates.

\section{APPENDIX Stimuli}

gels, pelk, fark, PELs; holk, dols, wers, DOLK; spen, spuk, brog, SPEK; rind, bund, dits, RUND; golk, tolm, kerg, TOLK; kalt, daft, polk, KAFT; klon, knos, prif, KLOS; hast, tasp, gond, TAST; plun, bluk, kren, PLUK; mijk, dijp, poeg, DIJK; kulp, gulm, penk, GULP; muls, ruts, zerm, MUTS; domp, poms, mels, POMP; wilp, nilg, sols, wILG; geuk, jeur, kuim, JEUK; slig, blib, wras, SLIB; derg, mirg, dups, MERG; vijk, mijg, kaan, vIJG.

Note-Nonword primes ( $\mathrm{SN}+, \mathrm{SN}-$, unrelated) are followed by target items (in capitals).

(Manuscript received November 22, 1999;

revision accepted for publication March 17, 2000.) 